\title{
iMedicalizar al delincuente o hacer del loco un criminal?: Prácticas de alienismo y medicina legal en Colombia a comienzos del siglo XX
}

\author{
Medicalizing delinquents or turning the mad into \\ criminals?: Practices of alienation and legal medicine in \\ Colombia in the early the 20th century
}

Manuela Barrios López ${ }^{1}$, Jorge Márquez Valderrama²

'Psicóloga. Becaria, Fundación Suramericana. Estudiante, Maestría en Historia, Universidad Nacional de Colombia, Medellín, Colombia. $\bowtie$ i

${ }^{2}$ Historiador. Doctor en Enseñanza y Difusión de las Ciencias y las Técnicas. Profesor titular Departamento de Estudios Filosóficos, Facultad de Ciencias Humanas y Económicas, Universidad Nacional de Colombia Medellín, Colombia. $\bowtie$
RESUMEN Este artículo analiza algunas asociaciones médico-jurídicas entre locura y criminalidad en el departamento de Antioquia (Colombia), en las primeras tres décadas del siglo XX. El análisis se orientó por dos ejes imbricados: el de los discursos y el de las prácticas. Se examinaron las ideas de cuatro médicos, planteadas entre 1917 y 1925, para identificar los debates teóricos desde los cuales se delimitaban y definían las enfermedades mentales en casos judiciales. La puesta en escena del saber de los peritos y su lugar como expertos se analizaron en un caso judicial, que inició en 1921, y en cuyo desarrollo afloraron las confrontaciones teóricas entre los médicos que debatieron sobre la posible locura del acusado. PALABRAS CLAVES Medicina Legal; Criminales; Medicalización; Simulación de Enfermedad; Trastornos Mentales; Colombia.

ABSTRACT This article analyzes medical-legal associations between madness and criminality in department of Antioquia (Colombia) during the three first decades of 20th century. The analysis was oriented by two overlapping axes: discourses and practices. The ideas of four doctors, generated between 1917 and 1925, were examined in order to identify the theoretical debates that delimited and defined mental illnesses in legal cases. The use of qualified knowledge and their place as experts were analyzed in a judicial case, initiated in 1921, in which theoretical confrontations surfaced among the doctors that debated the possible insanity of the defendant.

KEY WORDS Forensic Medicine; Criminals; Medicalization; Malingering; Mental Disorders; Colombia. 


\section{INTRODUCCIÓN}

La asociación entre locura y criminalidad ha sido abordada desde distintas direcciones, entre ellas, el estudio de los saberes y las prácticas que permitieron hacer del crimen un síntoma y del delincuente un enfermo ${ }^{(1)}$. Al respecto, en la producción iberoamericana (Chile, Argentina, México, Brasil y España) hay trabajos interesados por los procesos de recepción y apropiación de teorías que contribuyeron a concebir a ciertos locos como criminales y viceversa. Los autores coinciden en la relevancia que adquirió el delincuente como objeto de estudio desde distintas especialidades médicas como la psiquiatría, la medicina legal, la criminología y la psiquiatría forense, lo cual favoreció la construcción de un enemigo común con la validez de argumentos calificados como científicos ${ }^{(1,2,3,4)}$. Con el estudio de los casos judiciales, otros investigadores señalan los peritajes rendidos por expertos como instrumento de cientificidad y como una de las fuentes de legitimación del saber médico-legal(5,6).

La revisión bibliográfica también permite reconocer la vinculación tácita entre el comportamiento de ciertos individuos y el peligro que suponían para ellos mismos y para la sociedad. A partir de esta asociación se dieron procesos de estigmatización a grupos marginales (pobres, vagos, mendigos, inmigrantes) sobre los cuales se ejercieron prácticas de control con vistas a proteger el cuerpo social. En este sentido, también se señaló la existencia de prácticas de higienización social, así como acciones institucionales extendidas a grupos poblacionales considerados prejuiciosamente como "peligrosos"

En Colombia, el interés de distintos investigadores por la locura como realidad histórica, social y cultural tiene una trayectoria más bien reciente y los trabajos académicos en la materia parecen en auge en las últimas dos décadas ${ }^{(9,10,11,12,13,14)}$. Como punto en COmún, los investigadores coinciden en incluir la locura como parte de los distintos grupos de la población que implicaban un problema social (vagos, mendigos, pobres y delincuentes).
La locura se asoció a la ingesta de bebidas espirituosas (alcohólicas) ${ }^{(11,15)}$, a enfermedades como la sífilis ${ }^{(16)}$ y a situaciones sociales como la pobreza y la miseria ${ }^{(14)}$.

Entre las medidas de contención contra los alienados mentales, hubo inicialmente acciones de policía y luego de confinamiento $^{(17,18,19)}$. Las investigaciones centradas en las instituciones especializadas en el control de los locos reconocen que la creación de estos espacios tuvo objetivos más sociales que terapéuticos, como el de separar la locura de calles, cárceles y hospitales ${ }^{(12,13,17,18,19)}$. En este sentido, se entiende que el saber clínico sobre las afecciones mentales no fue consecuencia de la instauración de los espacios asilares.

Los procesos de profesionalización de especialidades médicas como la psiquiatría fueron $\operatorname{tardíos}^{(20,21,22)}$, pues incluso bien entrado el siglo XX solo unos pocos "Quijotes [...] se interesan por conocer la enfermedad mental y los enfermos mentales"(21). Algunos médicos interesados en la psiquiatría o la medicina legal fueron Guillermo Uribe Cualla, Luis Jaime Sánchez, Pablo A. Llinás, Carlos E. Putnam y Leoncio Barreto ${ }^{(23)}$. La vida y la obra de algunos de ellos fueron estudiadas por separado, como las de Miguel Jiménez López ${ }^{(24,25)}$, Edmundo Rico ${ }^{(26)}$ y Laurentino Muñoz Trujillo(27).

En las dos primeras décadas del siglo $X X$, la idea de una degeneración progresiva de la población colombiana se destacó, sobre todo, a partir de la discusión pública en los años veinte, en el seno de un grupo de intelectuales, liberales y conservadores: Luis López de Mesa, Miguel Jiménez López, Calixto Torres Umaña, Jorge Bejarano, Simón Araújo, Lucas Caballero, Rafael Escallón, entre otros ${ }^{(25,28,29,30,31)}$. El debate sobre la degeneración de la raza en Colombia fue interpretado por algunos investigadores como el reflejo de la acción directa del Estado para "impartir un nuevo orden a la sociedad desde su fundamento: el ciudadano"(30). En otra dirección, otros lo entendieron como el "debate de unos intelectuales colombianos, [...] que no solo no son miembros del gobierno, sino que son la mayoría de oposición al 
gobierno de Marco Fidel Suárez en 1920"(25). Más allá de las interpretaciones, quienes participaron en las conferencias dictadas en el Teatro Municipal de Bogotá en 1920, expusieron las causas y perspectivas del porvenir del pueblo colombiano al resaltar, en favor o en contra, los prejuicios basados en los determinismos biológicos y geográficos $^{(25)}$. En cuanto a la criminalidad, la metáfora del cuerpo humano y del organismo como vía de explicación del funcionamiento de la sociedad tuvo resonancia en la definición de términos médicos, en los intentos de explicación de la alta criminalidad y en la tipificación de ciertos delitos. La geografía y el clima de los territorios así como rasgos adquiridos mediante herencias degeneradas hicieron parte de las explicaciones causales de los actos en contra de la ley ${ }^{(29)}$.

Finalmente, algunos casos judiciales, célebres por el impacto que causaron en las sociedades de Medellín y Bogotá, han sido analizados especialmente. Entre ellos está el crimen del Aguacatal, en $1873^{(32)}$, el del Conde de Cuchicute, entre 1931-1938(33) , y el homicidio cometido por Jorge Zawadzky, en $1935^{(34)}$. En ellos la participación de médicos peritos y el lugar del dictamen médicolegal son leídos, bien como consecuencia del avance médico en asuntos legales ${ }^{(23,28,35)}$ o como instrumento para modificar el curso de los procesos jurídicos ${ }^{(34)}$.

\section{DEBATE CIENTÍFICO-MÉDICO SOBRE LA RESPONSABILIDAD LEGAL DEL DELINCUENTE}

El interés de la sociedad colombiana por el fenómeno de la locura en el siglo XIX se apoyó en el ámbito médico-legal. Esto quizá se debió a la cercanía que tuvieron los peritos médicos con algunas nociones psiquiátricas, al hacer reconocimientos del estado mental de algún individuo ${ }^{(23,36)}$. De ello da cuenta el hecho de que las primeras instituciones asilares especializadas en alienados mentales se organizaron a partir de 1870, mientras que la participación de médicos en juicios tuvo un recorrido anterior ${ }^{(17,18,19)}$. Si se comparan en secuencia diacrónica, los dictámenes referentes a la locura necesitaron una experticia cada vez más especializada en enfermedades mentales; no se trataba del trasplante de teorías o conceptos extranjeros, sino de una asimilación local de modelos foráneos en los que se dieron "reconceptualizaciones"(37). Se redefinieron, especificaron, reordenaron nociones, modelos, teorías, preguntas provenientes de diversos campos atraídos por la enfermedad mental. En las primeras tres décadas del siglo $\mathrm{XX}$, algunos médicos como Fidel Regueros, Hipólito Castro, José Eliseo Avellaneda, Primitivo Balaguera, Julio Ortiz y Luis Carlos Montoya, interesados en proponer modelos teórico-prácticos, publicaron en tesis y artículos sus ideas sobre la nosología psiquiátrica relacionada con el ámbito jurídico.

A finales del siglo XIX, en Colombia, en el estudio de los individuos prevalecían modelos tomados de las ciencias naturales orientados por diferentes tipos de determinismo, social, biológico y ambiental ${ }^{(38)}$, basados en el positivismo filosófico de Auguste Comte (1798-1857) y en el evolucionismo biológico de Herbert Spencer (1820-1903) (39). En medicina legal, estudios como el de Cesare Lombroso (1836-1909) y la Escuela positivista italiana contribuyeron a la medicalización del delincuente, pero también a señalar como criminales a ciertos pacientes. El médico italiano se había interesado desde muy temprano por la psiquiatría. Sus ideas fueron centrales en la configuración de una nueva perspectiva del saber sobre el delincuente que explicaba ciertas conductas criminales como productos de la constitución anatómica individual(40).

Por su parte, la teoría de la degeneración en la especie humana, propuesta por el alienista francés Bénédict Augustin Morel (18091873), condujo a establecer una diversidad de "anomalías" para identificar conductas potencialmente dañinas ${ }^{(41)}$. Morel llevó adelante sus estudios en París, donde mostró tempranamente sus intereses de investigación por las enfermedades mentales. Influenciado por las teorías evolucionistas para entender las relaciones entre la naturaleza y la sociedad, 
no abandonó su fe católica cuando propuso la idea de un "hombre de Edén" perfecto, original, del cual provenimos, pero con desvíos "enfermizos" transmitidos entre las genera-

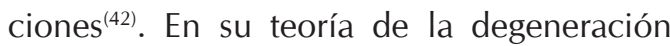
de la especie humana, se concentró en comprender las causas de la locura a partir de los factores hereditarios, restando importancia a los síntomas ${ }^{(40)}$.

Durante el proceso de institucionalización de la medicina legal en Colombia, modelos explicativos como el de Lombroso y el de Morel fueron parte de las disertaciones sobre las enfermedades mentales. Con ello se abrió la posibilidad de asumir ciertos comportamientos como parte de las alteraciones de la mente, sin que se tratara de un estado de completa insania.

El debate médico sobre los determinismos basados en la teoría de Lombroso generó críticas acerca de los modelos extranjeros sobre los que estaba fundamentada, los cuales no se acomodaban a los nacionales. Los doctores Antonio Blanco y Antonio Merlano refirieron en 1919 que los datos antropométricos europeos no eran aplicables a las condiciones etnológicas de Colombia, y consideraron más pertinentes "las taras hereditarias, con las manifestaciones del alcoholismo, del morfinismo, de la sífilis, de la epilepsia, de la tuberculosis"(43). En 1925, Guillermo Uribe Cualla persistía en esta crítica pues las descripciones sobre el criminal nato de Lombroso no eran homologables con los delincuentes colombianos:

\footnotetext{
...vemos caras comunes en nuestra raza india que, aunque atávicamente conserven las taras de sus ascendientes, sin embargo, son individuos que no habrían delinquido colocados en otro medio. ${ }^{(44)}$
}

Por otra parte, la locura entendida como sinónimo de sinrazón tuvo un punto de ruptura cuando se incluyeron las conductas anormales en el espectro de las alteraciones mentales ${ }^{(45)}$. El punto de discusión, en este caso, era la responsabilidad legal de las alteraciones graduales de la mente ${ }^{(44,46)}$. Al comparar las ideas publicadas por Uribe Cualla, en 1925, con las de Gil Juvenal Gil, de 1917, encontramos algunas coincidencias persistentes en el tiempo. Ambos autores compartían el interés por la medicina legal. Gil Juvenal Gil Madrigal (1884-1948), nacido en Yarumal, cursó estudios de medicina en Medellín, en la Universidad de Antioquia, pero también se graduó, en 1907, en la Universidad de Columbia de Nueva York. Su desempeño profesional se centró en gastroenterología, cirugía y ginecología, y fue docente de Patología Externa y Medicina Legal en la Universidad de Antioquia ${ }^{(47)}$. En cuanto a Guillermo Uribe Cualla (1895-1977), su grado lo obtuvo de la Facultad Nacional de Medicina de Bogotá, en 1920. Durante cincuenta años, trabajó en el Instituto de Medicina Legal de Colombia (antes Oficina Médico-Legal) y fue profesor de Medicina Legal en facultades de derecho y medicina en distintas universidades de

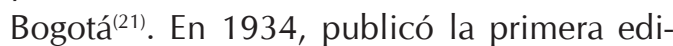
ción de su libro Medicina legal y psiquiatría forense, con múltiples reediciones, y creó, en 1953, la Revista de Medicina Legal de Colombia ${ }^{(23)}$.

En su artículo de 1917, el médico Gil Juvenal Gil reafirmó críticas que otros de sus colegas habían hecho antes ${ }^{(48,49)}$, una de ellas era que, en el lenguaje común y en el de los jueces, las palabras locura y demencia se usaban como sinónimos. Gil señaló que ese equívoco era propio de quienes se expresaban sin tener en cuenta la teoría médica que diferenciaba la demencia, o "debilitamiento general de todas las facultades mentales"(46), de la locura o "manifestaciones de palabra o acto de una enfermedad o defecto de la mente" ${ }^{\prime \prime(46)}$. Según Gil, entre sanos y enfermos existía "una gradación infinita de tipos que, partiendo del normal pasan por una zona intermedia"(46). A esto se añade, según él, que pocos individuos "son perfectos física y mentalmente, y todos cargamos con taras más o menos importantes" ${ }^{\prime \prime 46)}$.

Por su parte, Uribe Cualla refirió en su artículo que en la "prolongada escala entre el sér completamente normal [...] y el sér anor-

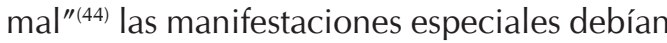
ser estudiadas a la luz del saber psiquiátrico para definir desde allí la responsabilidad de 
los afectados. Así, el tipo de "mentalidades intermedias" introdujo la idea del "anormal", que abarcaba individuos en los que las perturbaciones de la mente "no alcanzan los límites de la locura"(44).

En cuanto a la peligrosidad, ambos autores coincidieron en que los delincuentes sospechosos de anomalías mentales podían padecer eventos pasajeros, impulsivos, momentáneos, repetibles en el futuro, lo que dejaba a la sociedad en constante peligro y sumaba a estos estados una latente cronici$\operatorname{dad}^{(44,46)}$. Al respecto, la sugerencia de Gil era la reclusión de estos individuos en una institución especial, distinta al manicomio, y que fueran los médicos encargados quienes autorizaran la salida solamente por completa curación $^{(46)}$. En esta dirección, Uribe Cualla consideraba prioritario un establecimiento exclusivo para los "criminales anormales" que, por su "condición", eran "incapaces de vivir en la sociedad con los seres normales $^{\prime \prime(42)}$. Este tipo de asilos tendría una doble función: defender a la sociedad del peligro que representaban (excluirlos y contenerlos) $y$, al mismo tiempo, brindar un tratamiento científico a los internos.

En este sentido, cuando se trataba de determinar la responsabilidad jurídica de los acusados, el punto determinante para los médicos legistas era el examen sobre la conciencia y la voluntad, pues se trataba de facultades que podían estar obnubiladas, medianamente afectadas o completamente sanas como argumentaba Gil

...si del dictamen de peritos médicos legistas que han examinado detenida y cuidadosamente al criminal resulta que éste era un enfermo de la mente al momento del acto, ya sufra locura declarada o de estados patológicos que afecten temporalmente su razón, aunque esté sano en el momento del juicio, el juez está obligado moralmente a declararlo inocente del crimen. ${ }^{(46)}$

Uribe Cualla sostenía que las "doctrinas deterministas y las teorías lombrosianas" (44) no eran viables, pues desconocían muchos factores causales de los actos humanos. El perito debía tener presentes las múltiples causas que explicaban el comportamiento del delincuente, para discernir si las acciones delictivas eran producto de un estado de "anormalidad" pese a que, para el sentido común, se tratase de alguien "normal"(44). Un último punto discutido por estos autores médicos (Gil y Uribe CuaIla) fue el de la simulación, el engaño al que estaba expuesto el médico legista cuando un criminal "finge sufrir perturbaciones mentales o [...] el abogado que quiere valerse de este medio para salvar a su cliente" ${ }^{\prime(46)}$. Este último aspecto lo ilustramos seguidamente con un caso judicial.

\section{EL PERITAJE MÉDICO Y SUS ARGUMENTOS EN EL CASO DE UN POSIBLE SIMULADOR}

En el caso judicial se presentaron dos formas de explicar un mismo estado repentino de “alteración mental"(50), y uno de los puntos en los que se centraron las discusiones y las explicaciones fue precisamente la simulación. El expediente aporta los exámenes que efectuaron los peritos nombrados oficialmente así como los conceptos presentados como asesoría externa de los médicos del Manicomio Departamental de Medellín en 1924. Frente a las opiniones encontradas, el juez solicitó el acuerdo entre las partes para dar un veredicto final, pero las partes continuaron ampliando sus argumentos hasta el final.

Los hechos se presentaron el 24 de abril de 1921 en el Distrito de Andes (Antioquia) con una discusión entre los esposos Pedro y Magdalena. El altercado dejó de escucharse

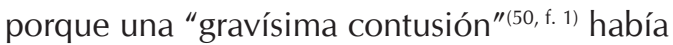
dejado inconsciente a Magdalena. Al día siguiente, la víctima fue trasladada al hospital de Concordia, pero murió al poco tiempo, habiendo alcanzado a señalar a su esposo como el agresor. Pedro fue interrogado en dos ocasiones: en la primera, negó cualquier disgusto con su mujer; en la segunda, admitió que ese día había llegado con hambre a casa pidiendo a su esposa que le diera de comer y que, en 
medio del pleito, la había sujetado por la garganta y la había lanzado contra la piedra de moler. Fue llevado a la cárcel de Andes (Antioquia) como posible autor del delito.

En la cárcel, sus compañeros de presidio se quejaron de la "enajenación mental" que Pedro exteriorizaba de forma violenta $y$, ante el peligro que representaba, pidieron que lo trasladaran al manicomio. Para ello, los peritos médicos Victoriano Toro E. y Arturo García lo examinaron y dijeron haberle hallado "diversos síntomas de enajenación mental". Describieron así su comportamiento: "meditabundo y abstraído", melancólico, agresivo, con "accesos de impulsividad" seguidos por periodos de "pasividad", con ideas "incoherentes", insomnio y "anorexia en veces" (50, f. 34). El informe de los peritos sugería su traslado con justa causa al manicomio departamental:

\begin{abstract}
...el Sr. Piedrahita no puede continuar haciendo vida común con los otros reos, en una cárcel tan incómoda y deficiente como la que hoy ocupa, [...] debe llevársele al Manicomio Departamental en donde no sólo reciba el tratamiento que su salud requiere sino que también será estudiado sistemáticamente por los médicos alienistas, quienes con los datos aquí contenidos y con los que obtenga del $\mathrm{Sr}$ Alcalde de Salgar podrán integrar su estudio y decidir de la responsabilidad moral y legal que le corresponda. ${ }^{\left(50,{ }^{\prime} .34\right)}$
\end{abstract}

El juez llamó a algunos testigos y solicitó la opinión de dos peritos oficiales para esclarecer el estado mental del procesado. Los testigos interrogados negaron cualquier síntoma de enajenación mental manifiesta en Pedro y en sus parientes:

Desde hace muchos años conozco a Pedro [...] jamás le he notado el menor síntoma de locura o demencia, por el contrario, siempre me ha parecido hombre muy cuerdo y muy alentado y en la familia de él no hay locos ni dementes todos ellos gentes cuerdas y sanas, pues ni aun alcohólicos, porque es gente que no usa jamás el licor. ${ }^{(50, \text { f. 36v) }}$
Es un hombre completamente cuerdo y muy sano, en la familia de este no hai ni ha habido locos ni dementes. ${ }^{(50, \text { f } .37-37 \mathrm{v})}$

Por su parte, quienes fungieron como médicos legistas, Jorge Sáenz y Luciano Restrepo, dictaminaron que, a partir de los testimonios del sumario, "Pedro estaba en pleno goce de sus facultades mentales en el momento en que ejecutó el acto criminal”(50, f. 40). Después de dirigirse al manicomio para examinarlo personalmente, estos médicos declararon la existencia de "varias anomalías congénitas, aunque no probablemente hereditarias" ${ }^{\prime \prime(50, \text { f. 40) }}$ ni suficientes para explicar la "rápida aparición de la enajenación mental pocos días después" de los hechos ${ }^{(50, \text { f. 40) }}$. Lo anterior generó dudas en los peritos sobre el estado de locura y dictaminaron que Pedro era, probablemente, un simulador.

Después de presentado el informe de los peritos y de haber visitado personalmente al acusado en el manicomio, el fiscal del caso solicitó una exposición de los médicos encargados de esa institución: Lázaro Uribe Calad y Bernardo Ferrer. El juez accedió a esta solicitud y descalificó el peritaje realizado por los médicos Sáenz y Restrepo, al calificarlo como "[sin] historia clínica alguna, ni razonamientos de carácter médico legal [...] falto de premisas científicas" ${ }^{\prime(50, \text { f. 41). }}$.

Uribe Calad, médico director del manicomio departamental, describió en la historia clínica el comportamiento de Pedro como retraído, con desorientación espaciotemporal, desnutrición avanzada, mutismo y

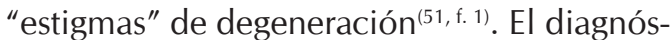
tico ajustado a su condición fue "melancolía aguda", en la que existían "periódicamente episodios de lucidez" que podían durar varios días ${ }^{(50, \text { f. } 44)}$. En el apartado de la historia clínica titulado "observaciones" se resaltaron las "respuestas incoherentes" acompañadas de algunos "momentos lúcidos" (énfasis de la fuente) ${ }^{(51, \text { f. 1) }}$, en los cuales Pedro hablaba de su vida pasada y de los sucesos fatídicos ocurridos en su hogar y cuyo desenlace fue la muerte de su esposa.

Los médicos Uribe Calad y Ferrer se opusieron a la idea de la simulación, aseguraron 
que Pedro, quien llevaba seis meses en el manicomio, era un enajenado. Consideraron suficiente este tiempo para su observación detenida y para concluir que no había nada de simulación. Resaltaron el informe de los médicos Toro y García (peritos reconocedores en la cárcel), que había permitido el traslado de Pedro al manicomio y servía como prueba de la alienación del sindicado. Realizaron un examen físico en el cual destacaron los estigmas propios de degeneraciones físicas como "la asimetría facial [...] la configuración especial de su rostro y de su cráneo, la exagerada longitud de sus miembros superiores en relación con la talla del individuo" ${ }^{\prime(50, \text { f. 42v). }}$. El comportamiento del examinado también revelaba su enfermedad. Toro y García se refirieron a estados intermitentes de conciencia y lucidez, en los que Pedro había expresado preferir ser "Ilevado al presidio donde tendría que llevar una condena y trabajar materialmente $^{\prime \prime(50, \text { f. 43) }}$. Esto último lo tomaron como indicio para dudar de la simulación, pues no era congruente que, si estaba fingiendo su locura, Pedro prefiriera la cárcel.

Aunque para Uribe Calad y Ferrer cabía la posibilidad de que no estuviera "enajenado" el día de los hechos, era necesario tener presente la degeneración "física y tal vez psíquica" f. 44) como predisponente de una impulsión homicida que, en este caso, lo había llevado a actuar como lo había hecho contra Magdalena. Además, el hambre podía sumarse como "factor de predisposición [...] a cometer un acto impulsivo"(50, f. 44v). Finalmente, sugirieron buscar antecedentes de ataques epilépticos y plantearon la siguiente pregunta: " $i$ El acto criminoso cometido por Pedro pudo ser la primera manifestación de una psicopatía que en estado latente existía en este individuo tan predispuesto a ella?"(50, f. 44v).

El fiscal recomendó al juez que, en el momento de la sentencia, tuviera en cuenta el informe presentado por "el connotado Dr. Bernardo Ferrer y por el eminente y renombrado profesor Dr. Lázaro Uribe [...] verdadera autoridad en la materia"(50, f. 45), máxime cuando ellos habían "tenido mayores y mejores oportunidades para examinar y observar al sindicado"(50, f. 45).
Ante las contradicciones de los informes periciales, "para unos un simulador para otros un enajenado"(50, f. 56), el juez rechazó por superficial el primer dictamen de los médicos peritos oficiales, Sáenz y Restrepo, y les solicitó que realizaran una nueva experticia ${ }^{(50, f .56)}$, pues su peritaje era el que tenía carácter de prueba legal, y no el de Uribe Calad y Ferrer, médicos del manicomio. Sáenz y Restrepo presentaron de forma individual la segunda opinión solicitada.

Por su parte, en el segundo informe Jorge Sáenz se ciñó al sumario. Tuvo en cuenta el cambio de versión entre la primera y la segunda indagatoria del reo, hecho que probaba lo "consciente, reflexivo y libre" de su estado el día de los hechos. La falta de antecedentes hereditarios de locura en la familia de Pedro le hacía suponer al médico Sáenz que las "anomalías" físicas eran "congénitas, pero no hereditarias; [...] motivadas por trastornos gestativos accidentales en la madre, sin que ni ésta ni el padre ni los ascendientes sufrieran estados morbosos transmisibles"(50, f. 50). Algunos testigos $^{(50, \text { f. 1, f. 3, f. 19, f. 16) }}$ dijeron que Pedro tenía celos de su esposa y que en el pasado ya se habían dado altercados entre los cónyuges. De ahí la imposibilidad de asumir lo acaecido el día de los hechos como una acción impremeditada por parte de Pedro, pues había obrado "en per-

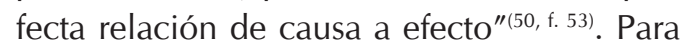
Sáenz, un loco actuaba sin motivo, guiado por una "idea fija, por una alucinación, por una ilusión, por una idea delirante o por un delirio completo" ${ }^{\prime(50, \text { f. 50), }}$, sin medir las consecuencias de sus actos, sin remordimiento. Sin embargo, para este médico, los hechos en cuestión no coincidían con esta descripción.

Sáenz no negó que Pedro estuviese "perturbado mentalmente", pero la coincidencia de la aparición de los síntomas en la cárcel, y no antes, se explicaba probablemente por el “cruel remordimiento" ${ }^{(50, \text { f. 50) }}$ y no por un estado previo de enfermedad mental. Al referirse a la "brillante exposición de los distinguidos

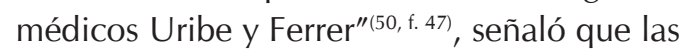
"anomalías físicas" no explicaban el estado mental de Pedro el día de los hechos. Aunque no se refirió al asunto de la simulación, 
aclaró que Pedro había sido consciente de sus acciones y por lo mismo era responsable de ellas $^{(50, \text { f. } 47) \text {. }}$.

La exposición de Luciano Restrepo complementó la de Sáenz con un tono reconocido por el juez como "irónico y airado [...] impropio en esta clase de asuntos" ${ }^{\prime(50, \text { f. 57). Aclaró }}$ que "sin ser especialista en enfermedades mentales se servirá de sus conocimientos en medicina" ${ }^{\prime(50, \text { f. 54) }}$ para anotar las contradicciones de sus colegas. Su informe se concentró en refutar una a una las conjeturas de ellos y cuestionó el profesionalismo de los alienistas al dejarse engañar por el reo. Uribe Calad y Ferrer dijeron que las palabras de Restrepo las tomaban como "brotes de ironía [...] falta de hidalguía profesional [...] fraseología intempestiva y tendenciosa"(50, f. 60).

Lo fundamental era averiguar, según la ley, el estado mental de Pedro el día de los hechos, pues eran "excusables y no están sujetos a pena alguna los que se hallen en estado de verdadera demencia o locura al tiempo de cometer la acción"(52). Aunque, los cuatro médicos que opinaron sobre el caso coincidían en el posible estado de alienación mental del acusado, el desacuerdo persistía en cuanto al tiempo de aparición de la enfermedad, situación que el juez necesitaba aclarar. El fiscal exigió que, dada la oposición entre los médicos (Sáenz, Restrepo, Uribe Calad y Ferrer), todos examinaran directamente al sindicado para elaborar un dictamen unánime.

El último concepto de los médicos del manicomio (Uribe Calad y Ferrer) se centró en defenderse de las críticas recibidas. Ratificaron y defendieron la exposición que habían hecho previamente. Explicaron que había "impulsiones psíquicas o de reflejo in-

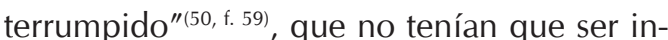
mediatas como había expuesto el Dr. Sáenz, caracterizadas por una lucha para inhibir el impulso en el interior "ideo-emotivo" (50, f. 59) de la persona; además el hambre definido

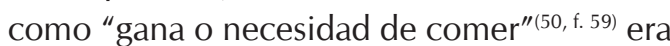
un estado fisiológico que podía llevar a "arrebatos impetuosos de ira" ${ }^{\prime(50, \text { f. } 60)}$ como podía haber ocurrido en el modo de actuar del acusado. El caso se cerró con la muerte repentina de Pedro en la institución asilar.

\section{CONSIDERACIONES FINALES}

Del estudio del expediente pudimos recrear parcialmente un escenario de prácticas donde se evidenciaron pugnas entre los peritos a partir de diversas estrategias de administración de la prueba. La discusión que se dio en esos primeros años del siglo XX nos permitió avanzar en algunas reflexiones sobre el lugar del saber de los peritos y la pregunta por la responsabilidad de los sospechosos de locura en casos como el presentado. Primero, está el valor que se le dio al peritaje, por encima de otros procedimientos probatorios como los testimonios, en los cuales se presentó unanimidad de los declarantes en cuanto a la ausencia de antecedentes de locura en Pedro. Segundo, los reparos del juez al primer peritaje oficial (Sáenz y Restrepo), por carecer de "bases científicas", revela en parte lo que se esperaba de este tipo de acción probatoria. Tercero, la idea del fiscal de considerar también la opinión de los médicos del manicomio puede indicar que los halagos y las sobreestimaciones fueron parte de las estrategias para orientar la sentencia en favor del estado patológico del acusado. En este caso se buscó una salida a las contradicciones de los dictámenes, mediante la ponderación de cualidades personales de los peritos, desde el renombre y prestigio de los mismos para descalificar o validar sus opiniones. Situación presente en otros casos judiciales en los cuales la simulación del sindicado llevó a los peritos a argumentar hábilmente para lograr convencer a las demás partes de la verosimilitud de su saber ${ }^{(53)}$ :

...los procedimientos para descubrir al simulador de locura dependen menos de la verdad sobre la enfermedad que de la suspicacia del médico. Lo que parece cierto, es que cada perito podía definir una serie de artimañas dependiendo del caso y del tipo de simulación sospechada. ${ }^{(53)}$

Los reconocimientos del estado mental de Pedro fueron objeto de debate. En las diversas 
posiciones se intentó argumentar desde el saber. Se identificaron distintas posturas científicas. Por ejemplo, en la consideración de los actos impulsivos entendidos por Sáenz y Restrepo como procesos automáticos e intempestivos, a diferencia de la opinión de Uribe Calad y Ferrer, que los explicaron como actos no necesariamente inmediatos en los que operaban luchas psíquicas internas.

Este proceso criminal diferenció dos terrenos de saber que podían estar presentes al mismo tiempo: el de los peritos oficiales, que en este caso fueron los médicos Sáenz y Restrepo, no como "especialistas" en enfermedades mentales sino como expertos o médicos legistas, y el concepto externo presentado por Uribe Calad y Ferrer, quienes de antemano contaban con la investidura de cientificidad que les otorgaban sus cargos respectivos. Las descalificaciones de los médicos legistas sobre los peritajes hechos por médicos de otros "ramos de la medicina" fueron una estrategia de apoyo a la idea de limitar esta práctica a los "especialistas". Al respecto, en 1931, Rafael Mejía calificaba de "extravagantes y anticientíficas [las] exposiciones hechas por médicos [...] de otros ramos de la medicina"(54). Así, la cientificidad se basaba en una suerte de grado de especialización del perito.

La apropiación local de teorías de la medicina occidental no fue homogénea por parte de los médicos que participaron en los asuntos médico-legales, en los cuales se debatía la definición de la locura. Entre los médicos identificados en este estudio, se pudieron observar puntos de vista eclécticos y flexibles, proclives a otorgar un criterio de cientificidad al peritaje demandado, como en el caso expuesto.

La entrada del peritaje en el andamiaje jurídico fue posible, en parte, por el cambio de postura de los jueces, quienes comenzaron a ver la locura como un estado patológico con implicaciones directas sobre la conciencia, la voluntad y el libre albedrío, conceptos trasversales al derecho. El perito médico entró como experto en la indagatoria a partir de su saber "científico", con el cual debía esclarecer las dudas de jueces, jurados y fiscales.
En casos judiciales como el presentado, el estatuto de verdad de los enunciadores estuvo atravesado por cualidades socialmente reconocidas que reforzaron el estatuto de veracidad de los dictámenes ${ }^{(45)}$. En cuanto al bagaje teórico y las controversias conceptuales sobre los delincuentes alienados, uno de los rasgos en común en las tesis y artículos de médicos colombianos de finales del siglo XIX y principios del siglo XX fue la amalgama de teorías causales sobre las enfermedades mentales en asuntos criminales y civiles.

La apropiación teórica tuvo matices en la flexibilidad de algunas nociones. Si bien los médicos fueron cuidadosos respecto de las ideas deterministas y materialistas en boga, estas alcanzaron una recepción relativa, y aunque fueron afines a relacionar la constitución anatómica o los factores fisiológicos con conductas patológicas, no se privilegiaba un único elemento etiológico (anatómico, fisiológico, hereditario, celular).

En el terreno jurídico, se definieron como "anormales" ciertas conductas, específicamente los actos atroces que suponían un desajuste interno, independientemente de la causa (psicológica, orgánica, neurológica, ambiental). Los médicos que consultamos reconocieron en sus escritos diversas causas que mediaban entre las acciones de los individuos $^{(44,46)}$. La ventaja de esta pluricausalidad de las perturbaciones mentales era la posibilidad de tener una incidencia directa sobre los factores predisponentes. En este sentido, ciertos determinismos podían servir de base a posturas pesimistas frente a la prevención y corrección de las enfermedades mentales, pero otras posiciones etiológicas fundamentarían cierto optimismo hacia una higiene que interviniera en lo social mediante la educación.

Así, la posibilidad de naturalizar el crimen llevó a que se considerara al delincuente como un objeto de estudio que demandaba un análisis de su constitución humana. Esto es evidente en el trabajo de los médicos peritos, "quienes tendrán que valorar no sólo la razón del sujeto sino también la racionalidad del acto, el conjunto de relaciones que ligan el acto con los intereses, los cálculos, el carácter, las inclinaciones, los hábitos del sujeto"(55). 
La pregunta por las relaciones entre saber médico y saber jurídico en lo concerniente a la locura sigue abierta, sobre todo por tratarse de realidades sociales dinámicas y sujetas a cambios en el contexto sociocultural, en los saberes, y en las prácticas sociales y profesionales. Este camino debe mantenerse, en tanto ayuda a comprender las formas de asir la locura como objeto de estudio desde criterios "objetivos" de la ciencia médica y jurídica, al tiempo que nos acerca a las rutas comprensivas de ciertos individuos en la realidad social.

Por último, en la redacción final de este resultado de investigación surge otra pregunta que queda planteada para futuros trabajos desde abajo, y que se refiere a la subjetividad de los señalados como dementes e infractores de la ley. En la búsqueda que se llevó a cabo, las explicaciones que los acusados, sindicados y sospechosos pudieran hacer de su propia situación no se vio expuesta en los expedientes consultados, y fueron los jueces, los médicos y los testigos quienes más información aportaron. En cambio, a los directamente responsables de los actos o a sus víctimas se les tuvo en cuenta más para el dictamen que para el esclarecimiento de los hechos.

\section{AGRADECIMIENTOS}

Este artículo es uno de los resultados de la investigación para tesis de Maestría en Historia "Locura y criminalidad en Antioquia. Conceptos de locura en sentencias y peritajes a finales del siglo XIX y comienzos del siglo XX" elaborada por Manuela Barrios y dirigida por Jorge Márquez Valderrama, integrantes del grupo de investigación Producción, Circulación y Apropiación de Saberes (PROCIRCAS) de la Universidad Nacional de Colombia. Se realizó con el apoyo financiero del proyecto "Medicalización de la infancia y de la locura examinadas a través de la institucionalidad asistencial y de la higiene mental en Colombia en el siglo XX", código HERMES 34773 coordinado por Jorge Márquez Valderrama y financiado por la Universidad Nacional de Colombia sede Medellín. Queremos reconocer el apoyo de la Fundación Sura, a través de la beca Nicanor Restrepo Santamaría, y agradecer el diálogo fecundo con los colegas de la Red Iberoamericana de Historia de la Psiquiatría (RIHP) y de la Red Colombiana de Historia de la Salud Mental.

\section{REFERENCIAS BIBLIOGRÁFICAS}

1. Campos Marín R. Crimen y locura: la patologización del crimen en la España de la Restauración. Norba-Revista de historia. 2007;(20):85-105.

2. Huertas R. Locos, criminales y psiquiatras: la construcción de un modelo (médico) de delincuencia. Átopos. 2006;(5):14-21.

3. Caponi S. Para una genealogía de la anormalidad: la teoría de la degeneración de Morel. Scientiae Studia. 2009;7(3):425-445.
4. Correa Gómez MJ. Cuerpo y demencia: La fisonomía de la incapacidad en Santiago de Chile (1855-1900). Historia Crítica. 2012;(46):88-109.

5. Ferla L. La pericia médico legal como forma de producir la verdad y sus adversarios en los tribunales brasileños de entreguerras. Asclepio. 2014;66(2):1-13.

6. Ruperthuz M, Sánchez M. Entre la degeneración y el psicoanálisis: una pericia médico legal chilena en 1915. Historia y Justicia. 2015;(4):138-168.

7. Campos Marín R. Higiene mental y peligrosidad social en España (1920-1936). Asclepio. 1997;49(1):39-59.

8. Rebelo F, Caponi S. A medicalização do crime: a Penitenciária de Florianópolis como espaço de saber e poder (1933-1945). Interface-Comunicação, Saúde, Educação. 2007;11(22):193-206.

9. Ospina M. "Con notable daño del buen servicio": sobre la locura femenina en la primera mitad del siglo XX en Bogotá. Antípoda. 2008;60(2):119-142.

10. Casas Orrego AL. Desplazamiento y aislamiento: Alienados mentales en la ciudad de Medellín 1879-1930. Asclepio. 2008;60(2):119-141.

11. Duque Ossa DJ, Quiceno Guzmán GC. Psicosis alcohólica en el Hospital Mental de Antioquia, 1900-1930. latreia. 2011;24(1):97-104.

12. Gutiérrez J, Marín Y. Poder psiquiátrico, formas clínicas y clasificación de la locura como enfermedad social, en el caso del Manicomio Departamental de Antioquia 1920-1959. Katharsis. 2012;(14):197-224. 
13. Rodríguez D. Los locos de Bogotá: del tratamiento y las representaciones de la locura en Bogotá, 1850-1930. [Tesis de grado]. Bogotá DC: Carrera de Historia, Facultad de Ciencias Sociales, Pontificia Universidad Javeriana; 2013.

14. Gutiérrez J, Márquez JH. Pobreza y locura como enfermedades sociales en la mentalidad civilizadora de la modernidad colombiana: Antioquia y Cundinamarca 1900-1960. Revista Facultad Nacional de Salud Pública. 2015;32(Supl 1):55-66.

15. Congote JC, Casas AL. Alcoholismo: enfermedad social en Medellín (1900-1930). En: Congote JC, Casas AL, (eds.). Salud y salud pública: Aproximaciones históricas y epistemológicas. Medellín: Hombre Nuevo Editores; 2013. p. 127-148.

16. Congote J, Casas A. Sífilis e higiene moral en la ciudad de Medellín: Observaciones clínicas de Cipriano Mejía Mejía a comienzos del siglo XX. En: Congote J, Casas A, (eds.). Actualizando discursos: Trazos de historia de la psiquiatría y de la salud pública en el contexto iberoamericano. Medellín: Facultad Nacional de Salud Pública de la Universidad de Antioquia; 2015. p. 275-293.

17. Montagut C. Formación del discurso psiquiátrico en Antioquia 1870-1930: una cartografía de la exclusión. [Tesis de maestría]. Medellín: Universidad Nacional de Colombia; 1997.

18. López Vélez L, Rueda D, Suárez Quiroz J. Historia institucional y terapéutica del hospital mental de Antioquia en sus 125 años. Revista Epidemiológica de Antioquia. 2007;29(1):9-123.

19. Silva J. Espacio, cuerpo y subjetividad en el Manicomio Departamental de Antioquia: 18751930. [Tesis de maestría]. Medellín: Universidad Nacional de Colombia; 2012.

20. De los Ríos A. Un siglo de psiquiatría en Antioquia 1878-1980. Boletín Comité de historia de la medicina. 1981;3(1):21-106.

21. Arteaga C. El ave fénix: Luis Jaime Sánchez Urrea (1916-1988). Revista Colombiana de Psiquiatría. 2001;30(2):179-198.

22. Gutiérrez J. Locura y sociedad: alienismo tardío, psicopatología e higiene mental en la modernidad colombiana, 1900-1968. [Tesis de doctorado]. Medellín: Universidad Nacional de Colombia; 2018.

23. Rosselli H. Historia de la psiquiatría en Colombia. T. 1. Bogotá: Editorial Horizontes; 1968.
24. Torres M. Un psiquiatra decimonónico en el siglo XX: Miguel Jiménez López (1875-1955). Revista Colombiana de Psiquiatría. 2001;30(2):113140.

25. Martínez A. La mayor controversia científica de la intelectualidad colombiana: Miguel Jiménez López y la Degeneración de la Raza (1913-1935). [Tesis de doctorado]. Tunja: Universidad Pedagógica y Tecnológica de Colombia; 2015.

26. Salamanca Rodríguez R. Con cetro de insigne marfil: Edmundo Rico Tejada (1899-1966). Revista Colombiana de Psiquiatría. 2001;30(2):141-160.

27. Vergara Suárez DM, Usuga Gutiérrez NB. La degeneración y la eugenesia en Colombia en textos de Laurentino Muñoz Trujillo 1930-1965. [Tesis de grado]. Medellín: Facultad de Ciencias Sociales y Humanas, Universidad de Antioquia; 2015.

28. Castañeda Medina SL. Una genealogía del racismo en Colombia: continuidades y discontinuidades del siglo XIX al XX. En: Castro-Gómez S, (ed.). Pensar el siglo XIX: Cultura, biopolítica y modernidad en Colombia. Pittsburgh: Instituto Internacional de Literatura Iberoamericana; 2004. p. 287-319.

29. Villegas Vélez A. Nación, intelectuales de elite y representaciones de degeneración y regeneración, Colombia, 1906-1937. Iberoamericana. 2007;7(28):7-24.

30. Pedraza Z. El debate eugenésico: una visión de la modernidad en Colombia. Revista de Antropología y Arqueología. 1997;9(1-2):115-159.

31. Muñoz Rojas C. Los problemas de la raza en Colombia; Más allá del problema racial: el determinismo geográfico y las "dolencias sociales". Bogotá: Editorial Universidad del Rosario; 2011.

32. Botero Bernal A. El crimen del Aguacatal: los relatos de causas célebres en el taller del iushistoriador. En: Mazzacane A, Conde E, Vallejo J, Botero Bernal A, Petit C. Causas célebres y derecho: estudios iushistóricos sobre la literatura, prensa, opinión pública y proceso judicial. Medellín: Universidad de Medellín; 2012. p. 93-137.

33. Rodríguez Gómez JC. El solitario; El conde de Cuchicute y el fin de la sociedad señorial (18711945). Bogotá: Universidad Externado de Colombia; 2003.

34. Ríos Molina A. Un crimen cometido en estado de ira e intenso dolor: Degeneracionismo y psiquiatría en la defensa de Jorge Eliecer Gaitán a Jorge Zawadzky, Colombia, 1935. Trashumante-Revista Americana de Historia Social. 2015;(5):38-59. 
35. Del Valle Montoya P. La medicalización de la justicia en Antioquia (1887-1914). Medellín: Editorial Universidad de Antioquia; 2010.

36. Valencia MFV. Degenerados, criminosos e alienados: Para uma história do conceito de degeneração na Colômbia, 1888-1950. [Teses de Doutorado]. Florianópolis: Universidad Federal de Santa Catarina; 2015.

37. Zuluaga Garcés OL. Pedagogía e historia: La historicidad de la pedagogía; La enseñanza, un objeto de saber. Bogotá DC: Foro Nacional por Colombia; 1987.

38. Speckman Guerra E. Crimen y castigo. Legislación penal, interpretaciones de la criminalidad y administración de justicia (Ciudad de México, 1872-1910). México DF: El Colegio de México, Universidad Nacional Autónoma de México; 2002.

39. García Ramírez CM, Giraldo Galeano SA. Esbozo de la apropiación política y jurídica del biologicismo determinista en la primera mitad del siglo XX en Colombia. Revista Prolegómenos-Derechos y Valores. 2015;18(35):81-102.

40. Postel J, Quétel C, (coord.). Nueva historia de la psiquiatría. México DF: Fondo de Cultura Económica; 2000.

41. Caponi S. Loucos e degenerados: uma genealogia da psiquiatria ampliada. Rio de Janeiro: Editora Fiocruz; 2012.

42. Conti NA. Benedict Augustin Morel y el origen del término demencia precoz. Vertex-Revista Argentina de Psiquiatría. 2003;13(53):227-231.

43. Blanco A, Merlano A. Apuntaciones médico legales sobre criminología en Colombia. Revista Médica de Bogotá. 1919;37(438-440):69-87.

44. Uribe G. Los criminales y su irresponsabilidad, bajo el punto de vista médico legal. Revista MédicoQuirúrgica de los Hospitales. 1925:239-244.

45. Foucault M. Los anormales: Curso en el CoIlège de France (1974-1975). Buenos Aires: Fondo de Cultura Económica; 2001.
46. Gil J. Nuestro código penal y la irresponsabilidad de los criminales por enfermedad mental. En: Segundo Congreso Médico Nacional de Colombia 1913. T. 3. Bogotá DC: Escuela Tipográfica Salesiana; 1917. p. 119-130.

47. Robledo E. La Medicina en los departamentos antioqueños. Repertorio Histórico-Academia Antioqueña de Historia. 1924;6(1-2).

48. Buendía N. Las monomanías impulsivas: Estudio clínico y médico-legal. [Tesis de doctorado]. Bogotá DC: Universidad Nacional de Colombia; 1893.

49. Castro H. Consideraciones generales sobre la demencia y las leyes. [Tesis de doctorado]. Bogotá DC: Universidad Nacional de Colombia; 1907.

50. Sección "Dementes e impedidos", Expediente 10373. Archivo Histórico Judicial de Medellín; 1921.

51. Historia clínica 5249. Archivo Histórico Judicial de Medellín; 1922.

52. Colombia. Ley 19 de 1890 (de 18 de octubre): Código Penal [Internet]. 1890 [citado 1 jul 2018]. Disponible en: https://tinyurl.com/y7xjpsk8.

53. Vásquez M, Caponi S, Gallo O. La simulación de estados patológicos mentales: Entre la psiquiatría y la medicina legal. En: Congote J, Casas A, (eds.). Actualizando discursos: Trazos de historia de la psiquiatría y de la salud pública en el contexto iberoamericano. Medellín: Facultad Nacional de Salud Pública, Universidad de Antioquia; 2015. p. $139-178$.

54. Mejía R. Práctica médico-legal. [Tesis de doctorado en Medicina y Ciencias Naturales]. Medellín: Universidad de Antioquia; 1931.

55. Foucault M. La evolución de la noción de "individuo peligroso" en la psiquiatría legal. En: Foucault M. La vida de los hombres infames. La Plata: Editorial Altamira; 1996. p. 54-68.

\section{FORMA DE CITAR}

Barrios López M, Márquez Valderrama J. ¿Medicalizar al delincuente o hacer del loco un criminal?: Prácticas de alienismo y medicina legal en Colombia a comienzos del siglo XX. Salud Colectiva. 2019;15:e1965. doi: 10.18294/ sc. 2019.1965

Recibido: 13 de julio de 2018 | Aprobado: 13 de septiembre de 2018 